\title{
Strategies for Radiology Reporting and Communication Part 3: Patient Communication and Education
}

\author{
Bruce I. Reiner
}

Published online: 10 October 2013

(C) Society for Imaging Informatics in Medicine 2013

\section{Introduction}

Changes in healthcare technology, economics, legislation, and societal expectations have collectively altered medical service delivery and communication. At the forefront of these changes is the patient who has become increasingly empowered and is now demanding greater access to their healthcare data and involvement in decision-making [1-3]. While radiology practice has traditionally limited communication of medical imaging report data between radiologists and referring clinicians, increasing emphasis is being placed on direct radiologistpatient communication, which has been mandated in breast imaging with the passage of the Mammography Quality Standards Act (MQSA) [4]. While radiology literature is replete with calls for obligatory communication of radiology report findings to patients [5-8], no practical solution has been offered to address the resulting workflow and workload challenges such communication requirements would entail if allocated to the interpreting radiologist. The existing communication paradigm used for MQSA mandates has largely delegated responsibilities to radiology department clerical staff, which in effect serve as proxies for the radiologist. The principle method of communication currently used is analogwith the use of standardized letters mailed to patients by certified mail (in order to document receipt), notifying them of the clinical significance and follow-up recommendations contained within the radiology report. While this approach serves to minimize radiologist workload, it does not address current economic, clinical, and educational deficiencies. If one was to attempt to expand the current radiology report communication model to include all medical imaging exams,

\section{B. I. Reiner $(\bowtie)$}

Department of Radiology, Veterans Affairs Maryland Healthcare System, 10 North Greene Street, Baltimore, MD 21201, USA

e-mail: breiner1@comcast.net existing deficiencies would become exponentially magnified and likely result in failure. The solution therefore is not to give up on the intended goal of improved and expanded patient communication, but instead creating a new communication model which effectively leverages computerized technologies for enhanced automation, data integration, operational efficiency, and workflow optimization.

\section{Current Communication Strategies}

Most existing patient communication models related to medical imaging exam findings rely on unidirectional text-based reports and letters (i.e., correspondence) to summarize pathologic findings contained within the radiology report along with follow-up recommendations. The content of these text-based reports or letters is largely fixed in nature, and customarily does not take into account the individual attributes of the recipient. The static nature of content is in part due to the fact that the communicating radiologist has minimal knowledge of the individual patient's educational background, medical knowledge, and clinical experience, along with the fact that customization is often time intensive and workflow inefficient.

Upon receipt of the written correspondence, the patient has a limited number of communication options. In the event that additional questions or concerns arise, the patient may elect to contact his/her primary care physician (or specialist) who is the physician responsible for ordering the medical imaging exam. In doing so, the patient will often seek clarification to unanswered questions or inconclusive information contained within the correspondence. The problem with this option is that it involves a third party who may or may not be fully aware of the correspondence in question, and may themselves have some degree of uncertainty related to its content. More often than not, the physician may not be the person directly addressing the patient concerns, but instead allocate these 
responsibilities to a staff member (e.g., nurse, aide), who is often not fully aware of the radiology report findings and/or the nuances associated with content contained in the correspondence. In addition to not completely addressing the patient concerns or understanding, this outsourcing of provider communication responsibilities (by either the physician or radiologist) has the potential to introduce uncertainty or even error, which becomes counterproductive to the intended communication objective or even harmful to patient outcomes.

If the patient is fortunate enough of directly addressing their questions or concerns with the physician or radiologist, the ensuing communication is typically done remotely, either over the telephone or electronically (e.g., e-mail). This often necessitates the physician/radiologist to retrieve clinical/ imaging data from the patient's medical record, which can be located in information system technologies such as picture archival and communication system (PACS), radiology information system, or electronic medical record (EMR). The relative lack of immediate data access will often lead to either communication time delays or incomplete data. Often times, the physician/radiologist will engage in the patient communication using the text-based radiology report alone, without direct review of the imaging dataset. The net result is that the ensuing remote patient communication is often limited in scope and in isolation to the imaging dataset, which arguably serves as the most valuable asset for patient communication and education.

The completeness of the patient communication is routinely defined by receipt acknowledgment of the correspondence (e.g., certified mail receipt). The assumption is made that the patient understands the content, criticality, and recommendations contained within the correspondence and will act accordingly. In most circumstances, however, no formal tracking is performed by the radiology service provider to ensure correspondence recommendations (e.g., biopsy, additional imaging study) were completed. The tracking of this data is difficult and cumbersome given the current communication model due to data nonstandardization and lack of integration. This places a great deal of trust and responsibility on the parts of the patient and referring clinician, which can result in unintended consequences. A preferable approach would be to incorporate automated tracking tools, which could provide automated prompts, alerts, and reminders of these recommendations to all communicating parties along with data integration to assess task completion and clinical outcomes.

\section{Defining Patient Roles, Responsibilities, and Preferences}

The mantra of any effective communication strategy should be "shared responsibility and accountability". To that end, as patients demand a greater role in their healthcare, they must also assume a greater amount of responsibility. As information is communicated between physician/radiologist and patient, it is incumbent upon both parties to ensure that the information shared is accurate, complete, comprehended, and acted upon. If for example, the radiologist communicates the urgency of a follow-up imaging exam and the exam is scheduled in a timely fashion, patient compliance is expected. At the same time, the imaging provider should take responsibility to ensure that the examination performed has been optimized to ensure quality, safety, and diagnostic accuracy. If the prior "paternalistic" approach of medical care providers is to be successfully transformed into a patient-centric approach fostering patient empowerment, patients must be willing and capable of assuming increased communication responsibilities.

Creating a standardized list of communication responsibilities and requirements is not practical however, given the reality that the heterogeneity of the patient population. A wide array of patient characteristics can be used to define patients into different profile groups (Fig. 1) which take into account differences in demographics, socio-economics, education, intelligence, computer proclivity, occupation, medical history, personality, and motivation. An effective communication strategy should take these unique patient attributes into account in an attempt to optimize clinical outcomes. The current "one size fits all" communication and technology models are unlikely to maximize success by not being adaptive and flexible to the needs, preferences, and abilities of the target audience [9]. This profile-based approach to patient communication can be used to customize communication content,
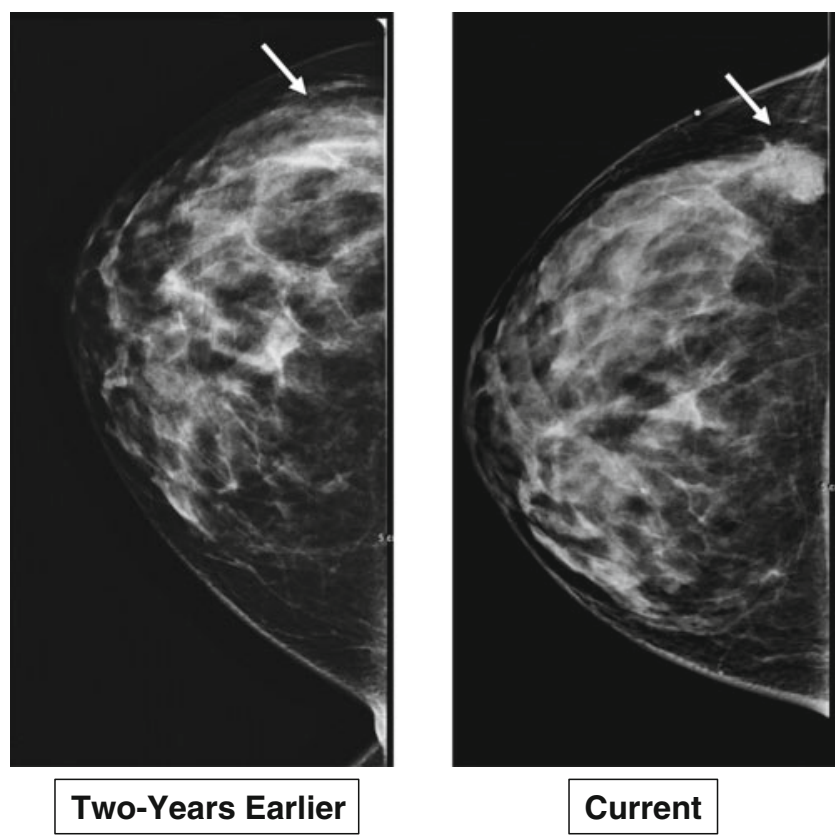

Fig. 1 Patient communication for newly diagnosed breast cancer on screening mammogram. Side-by-side sequential mammogram "key images" of the right breast are presented to illustrate a newly visualized mass in the lateral aspect of the right breast with arrows utilized to direct the attention of the reviewer to the region of interest 
delivery, and follow-up. As an example, a younger patient who is technologically savvy may prefer to have communication delivered electronically to their smart phone with regular updates, electronic scheduling, and video conferencing options along with context-specific Internet-based educational content downloads. An older patient who has not enthusiastically embraced computers may instead prefer to communicate in person, supplemented with mail correspondence. While one older patient may want to take a proactive role and be provided with the latest medical information regarding new technology and treatment options for a given disease, another may prefer to take a more passive role and simply be provided only with the "bare essentials". One patient may request that all communication be directed to them only, while another patient may request that a select group of family members and healthcare providers be included in correspondence (which can even be further customized in accordance with the specific context). The end result is that patient profiles and preferences can become effective tools for customizing communication schemas.

In addition to using these profile data and personal preferences to guide patient communication, another potential tool is the use of electronic auditing tools to optimize electronic communication in accordance with actual patient usage. Suppose for example, an electronic correspondence contains a number of context-specific links to Internet-based educational content. By auditing the usage patterns of an individual patient (as well as similar patients within their profile group) over time, predictive analytics can be used to predict the frequency with which content will be reviewed. In response to this data, providers (e.g., radiologists and clinicians) can modify the content options contained within future correspondences in keeping with the individual patient's usage history. At the same time, the cumulative frequency with which individual content options are utilized by large patient populations can serve as a barometer of perceived content quality, which can in turn drive future education content creation. Patients can also provide subjective feedback to further characterize their individual "likes/dislikes", which can serve as an additional tool for customizing correspondence and content options. The ultimate goal is to create an adaptive and flexible correspondence strategy which dynamically utilizes context and user-specific data to improve patient compliance, accountability, and outcomes.

\section{Image-Centric Communication}

While traditional radiologist-patient communication strategies are almost entirely text-based, a great deal of valuable data is being currently underutilized in the form of the medical imaging dataset. If a "picture is worth a thousand words", then why are the pictures (i.e., radiology images) not being taken advantage of for patient communication? The universality of visual imaging data (e.g., pictures and images) is of particular benefit, given language and educational differences among patients. It is far easier to show a patient a mass in an imaging dataset (along with complementary illustrative examples) than it is to try to explain it solely in words. At the same time, an oncology patient undergoing sequential imaging studies to monitor tumor burden during treatment can better comprehend tumor response by directly viewing select sequential images as opposed to being given numerical data alone. The goal therefore should be to create a communication method which utilizes both imaging and textual data in an attempt to improve clarity and comprehension. The use of imagery in patient communication also serves another important function in that the data is inherently standardized, which lends itself to creation of a referenceable database. This takes on importance as we begin to evaluate data mining applications and creation of best-practice guidelines.

The proposed communication strategy takes advantage of an existing application within most PACS, finding-specific key images. A number of limitations exist with existing strategies with regards to workflow, archival, data standardization, and integration. In the current PACS model of key image creation, the end-user (i.e., radiologist) must go through a series of steps in order to capture the key image from the comprehensive imaging dataset and must then manually input free text for annotation. The resulting "key image" is then stored in a separate folder from the primary imaging dataset, thereby requiring any subsequent end-user to manually open the key imaging folder for review. This is a distinct and separate process from the primary imaging dataset, which is automatically opened and displayed for review upon activation of the imaging exam. Once opened, the end-user is presented with the key image/s along with supporting text, which is distinct and separate from the corresponding radiology report. This relative lack of integration and nonstandardization of the supporting data results in a relatively inefficient process of data extraction and assimilation, which is counterproductive to the intended purpose of creating a shortcut (i.e., bookmark) of the most clinically important information contained within the imaging dataset. The end result is that existing workflow and data inefficiencies minimize the use and utility of key image functionality.

By understanding these existing deficiencies, we can devise a strategy of innovation which combines the theoretical benefits of key images with practical requirements of workflow optimization, data integration, and data standardization. One essential benefit of data standardization is the ability to customize presentation and content commensurate with the specific preferences and needs of individual endusers [10]. This ability to customize data takes on heightened importance when applied to the heterogeneous population of patients, which have marked differences in formal education, medical knowledge, clinical experience, and computer 
proclivity. One strategy to address this end-user heterogeneity is to create patient profiles which take into account a diverse number of variables (Table 1), and use these profile categories to create different versions of the technology in keeping with the attributes and skills of these different profile groups in the hopes of improving patient utilization and understanding. These patient profiles can be continuously updated to reflect differences in patient experience, knowledge, preferences, and use so that data customization and presentation would be dynamic in nature (show an example of how data can be presented differently for different patient profile groups). The customization of data according to different patient profile groups can extend beyond key imaging data and also be applied to supporting educational content.

The core information contained within the "key images" would consist of the images, standardized annotation and text, and links to related information. These links could consist of other imaging data within the patient imaging folder (e.g., key images from historical imaging exams), clinical data within the patient EMR (e.g., laboratory or pathology data), finding or disease-specific imaging data (e.g., central image database), or finding or disease-specific educational content (e.g., Internet or institutional patient education sites). In addition to providing education, the key images can also be used for a number of other functions including automated alerts and prompts (e.g., appointment reminders), longitudinal data tracking (e.g., follow-up clinical or imaging tests), and interactive consultations (e.g., radiologist-patient communications). The added value of using key images to support these functions is the concise and illustrative nature of the individually selected key images, the ability to integrate (i.e., superimpose) images with standardized annotation and text, and the ability to customize content and presentation in accordance with individual end-user profiles. Illustrative examples of how the proposed strategy would be used are presented in Figs. 1, 2,3 , and 4 . If a proverbial picture is worth a thousand words, then this approach of directly using imaging data for patient communication and education is far superior to the existing

Table 1 Characteristics and attributes used in creation of patient profile groups

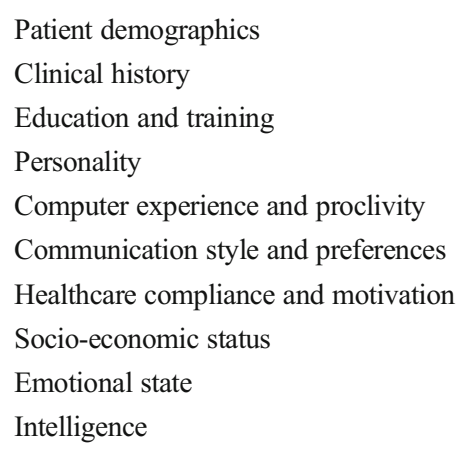

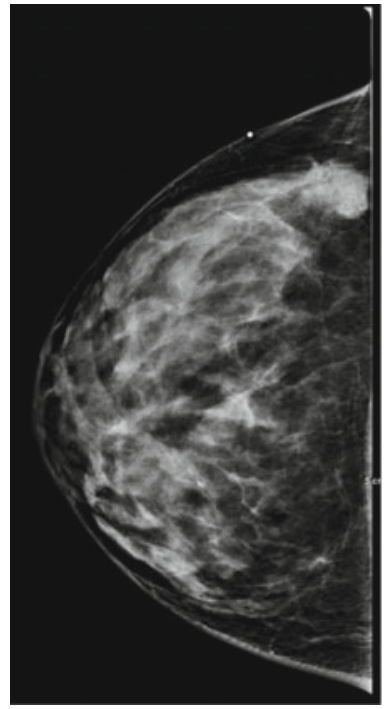

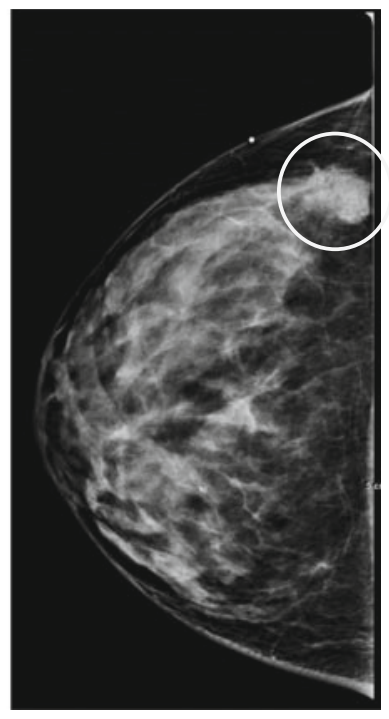

$$
\begin{aligned}
& \text { Diagnosis: Breast Cancer } \\
& \text { Clinical Significance: High } \\
& \text { Follow-Up Recommendations: Biopsy }
\end{aligned}
$$

Fig. 2 Annotation of key image with linked supporting data. Images of the right breast mass are presented with and without annotation (circle) of the mass in question. When the reviewer highlights the annotated region of interest, a box is automatically opened which contains fundamental data related to the diagnosis (or differential diagnosis), clinical significance, and follow-up recommendations associated with the finding of interest

model of narrative-free text reports, which are static, inflexible, and disassociated from the core imaging data which they are reporting on.

\section{Redefining the Radiologist-Patient Relationship}

In contemporary practice, radiologists are being constantly challenged by economic, geographic, technical, clinical, and societal pressures. In addition to increasing commoditization pressures, radiologists must deal with the reality that unlike their clinical colleagues, radiologists have little to no relationship with the patients they provide services to. As a result, most radiologists do not have the opportunity to create goodwill with the patients and provide a means with which they can foster a long-term and synergistic relationship. Historically, the principle ways in which radiologists circumvented this lack of patient familiarity and mutual commitment was through geographic, institutional, and physician protective boundaries. Most radiologists worked under the protection of the institution in which they were guaranteed exclusivity on all imaging studies performed at the institution in which they practiced. At the same time, patients tended to follow geographic boundaries and select local imaging service providers for their medical imaging needs. The physicians serving on staff at these institutions developed close working 
Fig. 3 Customizable patient education for newly diagnosed breast cancer. In order to provide customizable patient educational content, a patient profiling schema is used for patient categorization. On a simplistic level, this profiling schema can divide patients into three basic groups (basic, intermediate, and advanced) with the resulting content options commensurate with the individual patient's profile. In example $\mathrm{A}$, the patient has been classified into the "basic" educational profile with Spanish as the primary language. A video was incorporated into the content options in keeping with patient preferences. In example $\mathrm{C}$, the patient was classified into the "advanced" educational profile group, with resulting content and educational links designed to convey more detailed data, inclusive of statistics

Group A: Basic Content, Spanish

Basic facts: Family Medicine Perspective

http://www.familymedicineassociates.com/important-facts-about-breast-cancer

Diagnosis and treatment: Medline Plus (Spanish)

http://www.nlm.nih.gov/medlineplus/spanish/breastcancer.html

Video for Basic Understanding (Spanish)

http://www.youtube.com/watch?v=91leCnJuzHU

Group C: Advanced Content, English

Breast Anatomy

Types of Cancer

Hereditary and Risk Factors

Treatment Options

Statistics

Memorial Sloan Kettering Cancer Center

http://www.mskcc.org/cancer-care/adult/breast/patient-education

National Cancer Institute

http://www.cancer.gov/cancertopics/types/breast

Susan G. Komen Breast Cancer Foundation

http://ww5.komen.org/breastcancer/understandingbreastcancer.html

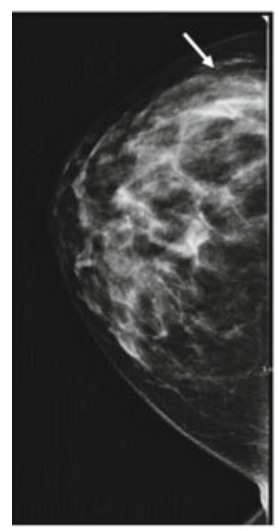

Initial negative mammogram minus 2 years

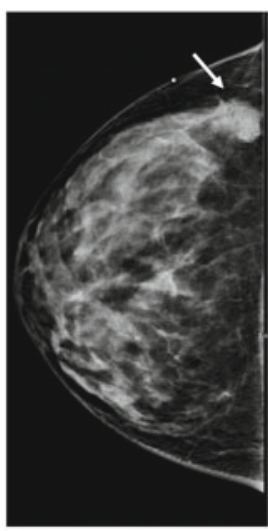

Initial diagnosis of cancer times 0

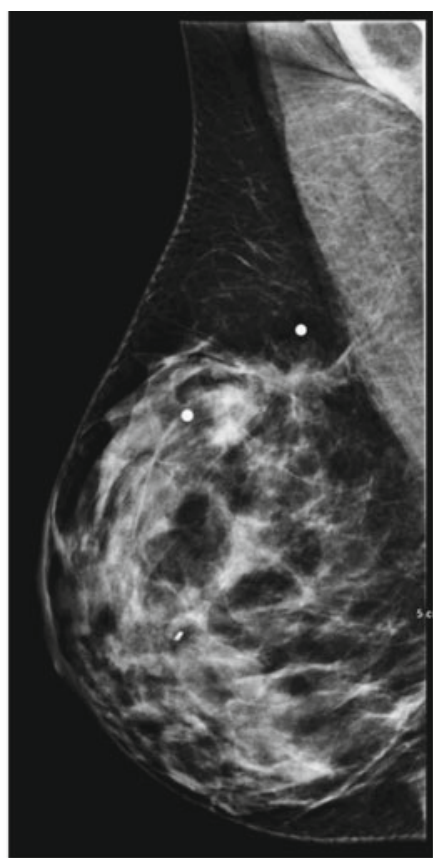

Cancer-free

Treatment: surgery/radiation +1.5 years 
relationships with the radiologists, which in turn created a sense of familiarity, confidence, and loyalty in referring their patients to these radiologists.

Over time, many of these protective mechanisms began to disappear with changes in technology (i.e., PACS and the digitization of medical imaging), outsourcing of professional radiology services (i.e., teleradiology), imaging workflow (i.e., remote and ubiquitous accessibility of medical images), and economics (i.e., physician self-referrals of imaging services). As radiologists struggle to maintain their economic and professional viability, they realize that establishing and fostering continual and long-lasting relationships to patients is fundamental to their continued success and professional survival. While the notion of creating "added value" to medical imaging services is commonly entertained (references), few tangible and practical solutions currently exist for the workflow-challenged community of private practice radiologists. The proposed innovation attempts to create a solution to these challenges by expanding the role of the radiologist from that of an expert interpreter of medical imaging studies to one of patient advocate, educator, and consultant. Radiologists have a fundamental and arguably unique expertise on issues related to medical imaging safety, quality, and economics. This expertise has been largely marginalized in conventional practice with radiologists largely excommunicated by the patients they serve. The creation of an image-centric tool to facilitate patient communication, education, and consultation should lie at the center of any "value added" strategy aimed at enhancing the perceived value of radiologists. At the same time, time constraints severely handicap the ability of radiologists to engage in person-to-person dialogue with patients, thereby necessitating the creation of electronic tools to facilitate customizable and interactive communication strategies.

In addition to fostering long-term patient loyalty through service differentiation, the proposed model also has the potential to create new economic opportunities for radiologists offering expanded patient services. While the current reimbursement model does not provide a direct economic incentive to provide these "added value" services, it is not inconceivable that additional reimbursements cannot be provided in the future. The "boutique model" primary care delivery has shown that affluent patients are frequently willing to pay a premium to those physician providers offering expanded and customized services. This model could in theory be extrapolated to radiology service providers who could offer a suite of consultation and educational services which go beyond professional interpretation alone. At the same time, as third-party payers continuously strive to enhance clinical outcomes through improved utilization, patient safety (e.g., radiation dose reduction), enhanced communication, and patient education, it is not beyond the realm of possibility that an expanded reimbursement model could result. In addition to the potential economic incentive provided to the consulting radiologist, patients themselves could derive economic benefit from active collaboration in these proposed education, communication, and consultation services through reduced medical insurance premiums.

The core of any potential changes in economic reimbursement should be objective outcomes analysis, which would require clinical research to substantiate that the proposed innovation would have a positive effect on clinical outcomes. Numerous examples exist in the medical literature which demonstrates the clinical improvements gained through enhanced patient communication and education [11-13], which up to now have largely ignored the role of radiologists. The time is right for the radiologist community to embrace an expanded role as patient advocate, educator, and consultant. Doing so, however, requires innovation and the simplest place to start is with the medical image itself.

\section{References}

1. Paglieri C, Detmer D, Singleton P: Potential of electronic personal health records. BMJ 335:330-333, 2007

2. D'Alessandro D, Dosa NP: Empowering children and families with information technology. Arch Pediatr Adoles Med 155:1131-1136, 2001

3. Woolf SH, Chan ECY, Harris R, et al: Promoting informed choice: transforming healthcare to dispense knowledge for decision making. Ann Intern Med 143:293-300, 2005

4. Linver MN, Osuch JR, Brenner RJ, Smith RA: The mammography audit: a primer for the Mammography Quality Standards Act (MQSA). AJR 165:19-25, 1995

5. Berlin L: Communicating results of all outpatient radiologic examinations directly to patients: the time has come. AJR 192:571-573, 2009

6. Berquist TH: Communication: the needs of the patient come first. AJR 192:557-559, 2009

7. Hall FM: The radiology report of the future. Radiology 251:313-316, 2009

8. Smith JN, Gunderman RB: Should we inform patients of radiology results? Radiology 255:317-321, 2010

9. Reiner B: One size (doesn't) fit all. J Am Coll Radiol 4:567-570, 2008

10. Reiner B: Customizing medical data. J Digit Imaging 23:363-373, 2010

11. Bodenheimer T, Lorig K, Holman H, et al: Patient self-management of chronic disease in primary care. JAMA 288:2469-2475, 2002

12. Stewart M, Brown JB, Donner A, et al: The impact of patientcentered care on outcomes. J Fam Pract 49:796-804, 2000

13. Leong SL, Gingrich D, Lewis P, et al: Enhancing doctor-patient communication using e-mail: a pilot study. J Am Board Fam Med $18: 180-188,2005$ 